\title{
SUGAR AND AGGRESSIVE AND INATTENTIVE BEHAVIOR
}

The effect of a sucrose challenge on aggressive behavior and sustained attention in a sample of young hyperactive boys and age-matched control subjects was studied at the Schneider Children's Hospital, Long Island Jewish Medical Center, New York, and Albert Einstein College of Medicine, Bronx, New York. The index group met DSM-III criteria for ADD-H and oppositional disorder as determined by interviews using the diagnostic interview for children and adolescents. They also scored at least 14 or above on the hyperkinesis index of parent and teacher revised questionnaires. Baseline testing was followed by a breakfast containing $8 \mathrm{oz}$ of an orange colored and flavored drink containing either $35 \mathrm{~g}$ of sucrose or adjusted to equal sweetness with either saccharine $(175 \mathrm{mg})$ or aspartame $(175 \mathrm{mg})$. The sugar and placebo challenges were given with a breakfast high in carbohydrate. There were no significant effects of sugar or placebo on the aggressive behavior of either group. Inattention measured by a continuous performance task increased in the ADD-H group following sugar, but not with saccharine or aspartame control. (Wender EH, Solanto MV. Effects of sugar on aggressive and inattentive behavior in children with attention deficit disorder with hyperactivity and normal children. Pediatrics Nov 1991; 88:960-966.) (Reprints: Dr. Wender, Schneider Children's Hospital, New Hyde Park, NY 11042.)

COMMENT. A deterioration in attention following sugar ingestion has been reported in one other study by Connors et al. in which sugar was administered with a high carbohydrate breakfast. When a negative effect was observed in 3 other studies the sugar was given either in a fasting state or with a protein breakfast. Further studies are indicated.

\section{HEAD SIZE, IQ AND BIRTH WEIGHT}

Head growth and IQ were correlated at ages 2, 5 and 8 years in children grouped according to birth weight in the Departments of Obstetrics and Gynecology and Pediatrics, University of Melbourne, Australia, and the Royal Women's Hospital, Melbourne. All children were white and with no signs of moderate or severe cerebral palsy. Using the National Center for Health Statistics reference values more group 1 children (birth weight 500-999 g) than group 2 children (birth weight 1,000-1,499 g) were below the 10 th percentile at ages 2 and 8 years. Using the Nellhaus standards the difference between groups 1 and 2 was not significant and head growth appeared to be satisfactory at age 2 years. Comparing group 3 (birth weight greater than $2,500 \mathrm{~g}$ ) with groups 1 and 2 combined, fewer of the group 3 children were below the 10 th percentile at both ages 2 and 8 years using the NCHS reference and at age 8 years using the Nellhaus data. The heads of very low birth weight children were progressively narrower but not longer than those of normal birth weight children. Dolichocephaly was unrelated to IQ and the correction of occipitofrontal circumference for dolichocephaly was rarely of clinical importance. Occipitofrontal circumference best correlated with the full scale IQ on the Wechsler Intelligence Scale for Children-Revised. At age 8 years the Wechsler IQ was significantly related to the OFC ratio in both 
very low birth weight and normal birth weight children. The AP-BP ratio was unrelated to IQ (Kitchen WH et al. Very low birth weight and growth to age 8 years II: head dimensions and intelligence. AJDC Jan 1992; 146:46-50.) (Reprints not available.)

COMMENT. The authors concluded that the National Center for Health Statistics data for occipitofrontal circumference measurements were more appropriate than the Nellhaus data in this study. Head growth achieved by age 8 years was progressively reduced in lower birth weight categories in all measurements except the anteroposterior diameter. Occipitofrontal circumference was closely related to IQ and other head measurements are not recommended in routine clinical practice. In a further report the authors found that maternal height and the birth weight ratio were more important than health after birth in predicting a height or weight below the 10 th percentile at age 8 years in children with low birth weights. Occipitofrontal head circumference is related to infant development and later intelligence in childhood and is a useful indication of brain size.

\section{CEREBRAL PALSY}

\section{ANTEPARTUM CAUSES OF CEREBRAL PALSY}

Six neonates with destructive brain lesions of fetal onset, diagnosed by radiological and neurophysiological studies, are reported from the Departments of Pediatrics, Neurology, Radiology, and Obstetrics and Gynecology, Magee-Womens Hospital, Pittsburgh, PA and Children's Hospital of Pittsburgh. Initial diagnosis of cerebral lesions was made by fetal sonography in 2 patients and CT scan in 4 during the first 30 hours of life. No intrapartum difficulties were noted and 2 patients had definitive evidence of maternal and placental disease that preceded the onset of active labor. The neurological examinations were normal at birth, but 4 presented with isolated seizures at 8-30 hours of life and initial neonatal EEGs showed abnormalities. The authors recommend that cranial imaging and neurophysiological studies should be used during the first days of life for neonates believed to have cerebral lesions based on maternal sonography or isolated seizures. (Scher MS et al. Destructive brain lesions of presumed fetal onset: antepartum

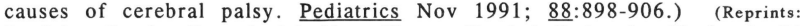
Dr. Scher, Develop Neurophysiol, Magee-Womens Hospital, Forbes St., Pittsburgh, PA 15213.)

COMMENT. Cranial ultrasonography and CT studies during the first few days of life may document lesions occurring prenatally. Children with antepartal brain injury may be asymptomatic or exhibit few clinical signs during the neonatal period and may later develop cerebral palsy.

\section{MRI IN ATHETOTIC CEREBRAL PALSY}

The MRI in 22 children with athetotic cerebral palsy was studied in the Department of Pediatric Neurology, Seirei-Mikatabara General Hospital, 